\title{
Using MBED to check Heart Beats
}

\author{
Li Duan ${ }^{1, a}$ \\ ${ }^{1}$ Glasgow College, University of Electronics Science and Technology, Chengdu 611731, China \\ a2014200101035@std.uestc.edu.cn
}

Keywords: MBED;Heart Beats;NXP LPC1768;Ratio

\begin{abstract}
In this project, the mbed NXP LPC1768, the sensor TCRT 1010, and the LED Driver Max7219, LED screen and the PCB are used to show the pulse. The optical sensor can measure the pulse when a finger is put on it. The flowing blood in our fingers can reflect the light back to the sensor and different velocity of the blood means different pulse and will lead to different output voltage signal of the sensor so that we can measure our pulse. A circuit is built to be a filter and the mbed is used to analysis the data and display the ratio and the shape of the pulse.
\end{abstract}

\section{Introduction}

Measurement of heart rate and observation on electrocardiogram (ECG) have been widely used not only in the field of medical treatment but also in the public' $s$ daily lives ${ }^{[1-3]}$. People sometimes need to detect their heart rate, for example, while long-distance running, to ensure their bodied are in safety $^{[4]}$. In order to achieve this, a kind of portable device is needed ${ }^{[5]}$. This project is done for a second year team design course and it aims to design and test a pulse meter to simply show a man' $\mathrm{s}$ heart rate and ECG on two $8 * 8$ LED screens simultaneously by detecting his blood flow with putting one of his fingers on an optical sensor.

\section{Overall diagram}

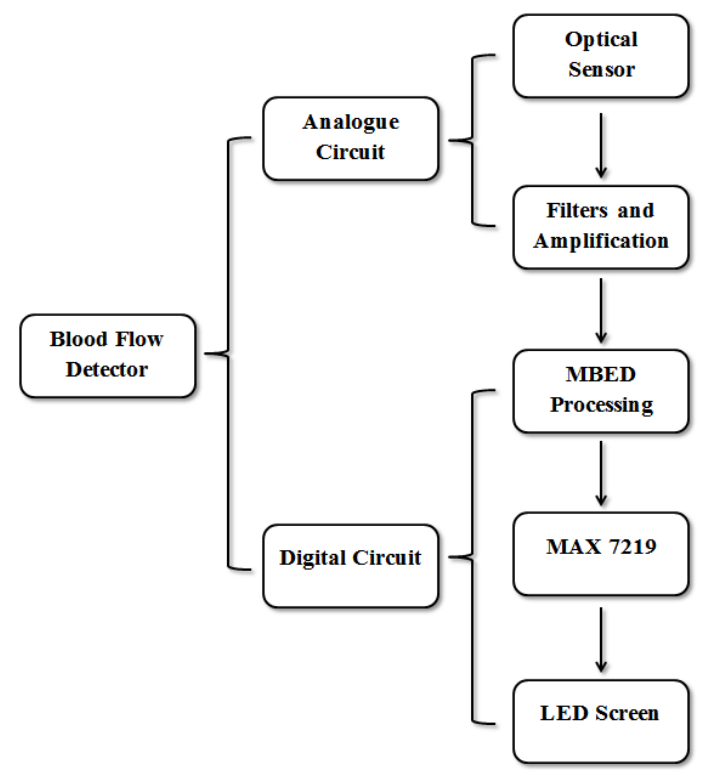

Fig. 1 the block chart of the whole project

\section{Specification}

The design of this project can generally be divided into two main parts, analogue circuit and digital circuit respectively.

The purposes of analogue circuit part: 

$5 \mathrm{~V}$

powering up the whole circuit by using a pressure regulator ( $\mathrm{L} 78 \mathrm{~S} 05 \mathrm{CV}$ ) to change $9 \mathrm{~V}$ (battery) to

getting signals of blood flow from one man's fingers by using an optical sensor (TCRT1010)

Filter the noise not wanted because what matters is the signals of $0.1 \mathrm{~Hz}$ to $10 \mathrm{~Hz}$

amplifying the signal ( $A v \approx 750$ ) by using two active filters (LM324)

transferring the filtered and amplified signal to MBED for next processing

The purposes of digital circuit part:

processing the signal with the coed compiled in MBED (ADC and SPI)

Ttransfer the signal to MAX7219, the driver of the $8 * 8$ Nixie.

displaying heart rate and electrocardiogram on the LED screen

\section{Main part of the hardware}

Sensor:
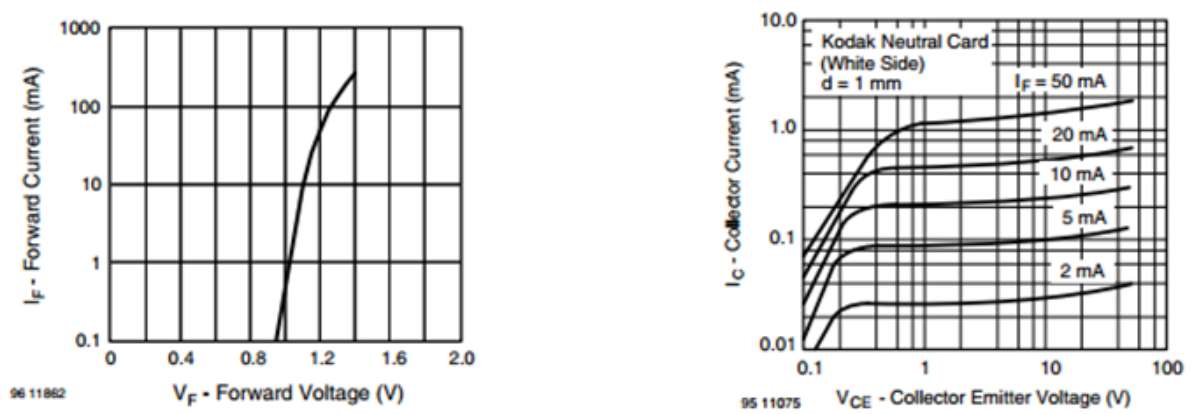

Fig. 2 the relationship between the collector current and collector emitter Voltage

With the datasheet of the optical sensor TCRT1010, the diode and the detector need suitable and stable current, the resistance $91 \Omega$ and $10 \mathrm{~K} \Omega$ were chosen for them respectively.

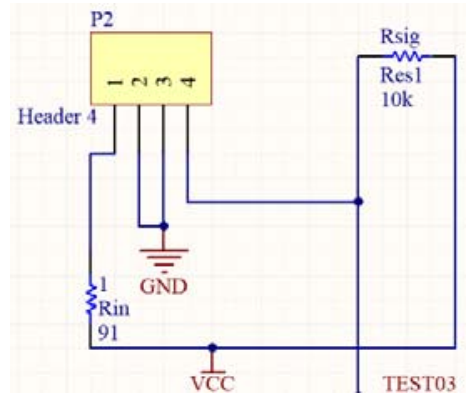

A. Amplifier:

Fig.3 the circuit for the optical sensor

$$
\begin{gathered}
f_{H}=\frac{1}{2 \pi R_{H} C_{H}}=10 \mathrm{~Hz} \\
f_{L}=\frac{1}{2 \pi R_{L} C_{L}}=0.1 \mathrm{~Hz}
\end{gathered}
$$

According to the equation of the cut off frequency. Choose the resistance $1 \mathrm{M} \Omega$ and the capacitor $1 \mathrm{uF}$ for the high-pass filter. And resistance $16 \mathrm{~K} \Omega$ and capacitor $1 \mathrm{uF}$ for the low-pass filter. 


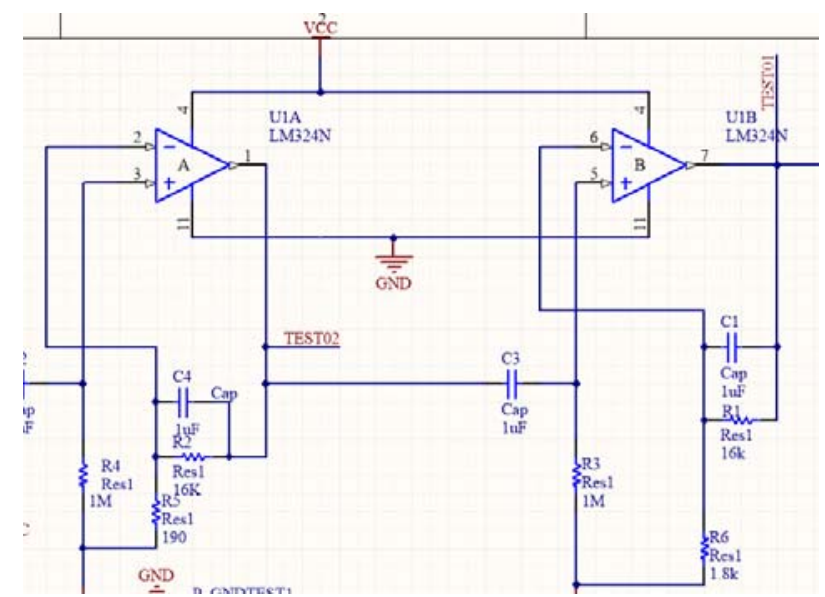

Fig.4 the two stage of the amplifier circuit

In the circuit, the active amplifier is used to amplify the signal, the original signal is about $5 \mathrm{mV}$ and the gain of the amplifier is about, so the output signal is about $3.75 \mathrm{~V}$.

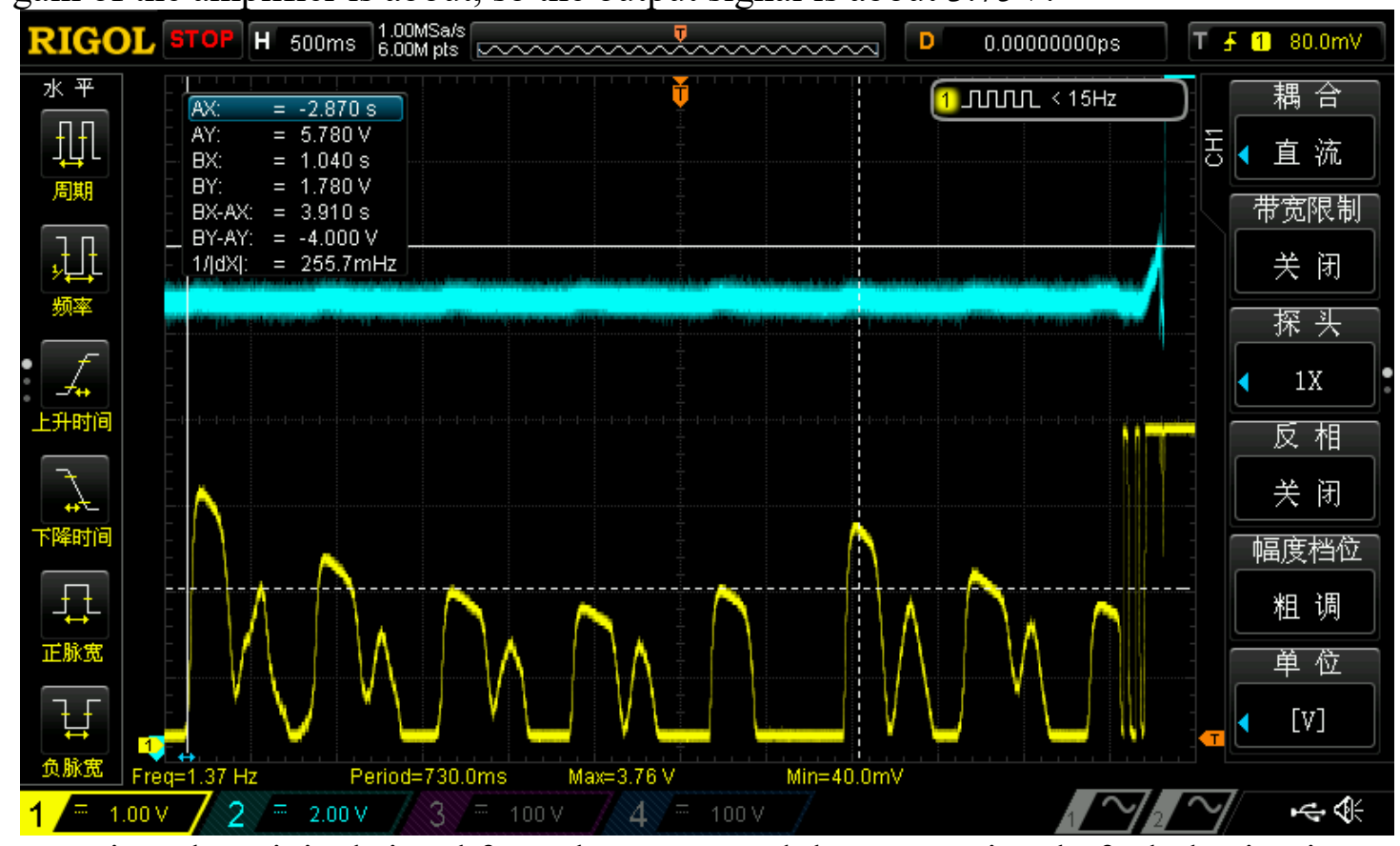

Micro:

Fig.5 the original signal from the sensor and the output signal of whole circuit

The freedom board in this project is very important. Because it is very convenient to adjust or change the component on the freedom board.

About the microcontroller, the NXP LPC1768 is used because its online compiler is very convenient and the open library is useful for the code writing.

PSU:

As the amplifier and the sensor need a $5 \mathrm{~V}$ power supply to work, but the output voltage of the battery is about $9 \mathrm{~V}$. So the circuit need a PSU which can decrease the voltage to $5 \mathrm{~V}$ so that is fitful for the amplifier and the sensor. There are two capacitor was connected to the in/out port of the regulator, is used to stabilize the voltage. 


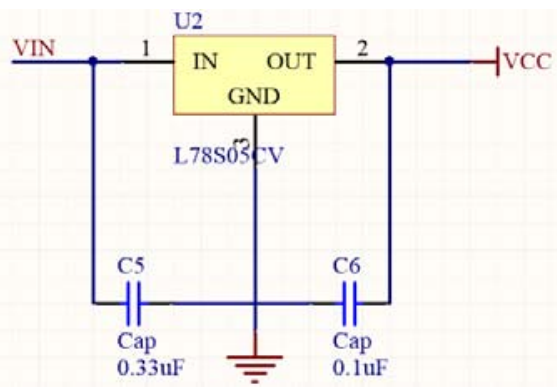

Fig. 6 the circuit for the PSU. VIN $=9 \mathrm{~V}$ and $\mathrm{VCC}=5 \mathrm{~V}$

But with the battery, the circuit only works about 2 hours, and the voltage of the battery will drop slowly. So the mbed $5 \mathrm{~V}$ output is use in this circuit to be an alternative power supply system.

\section{Figures and waves}

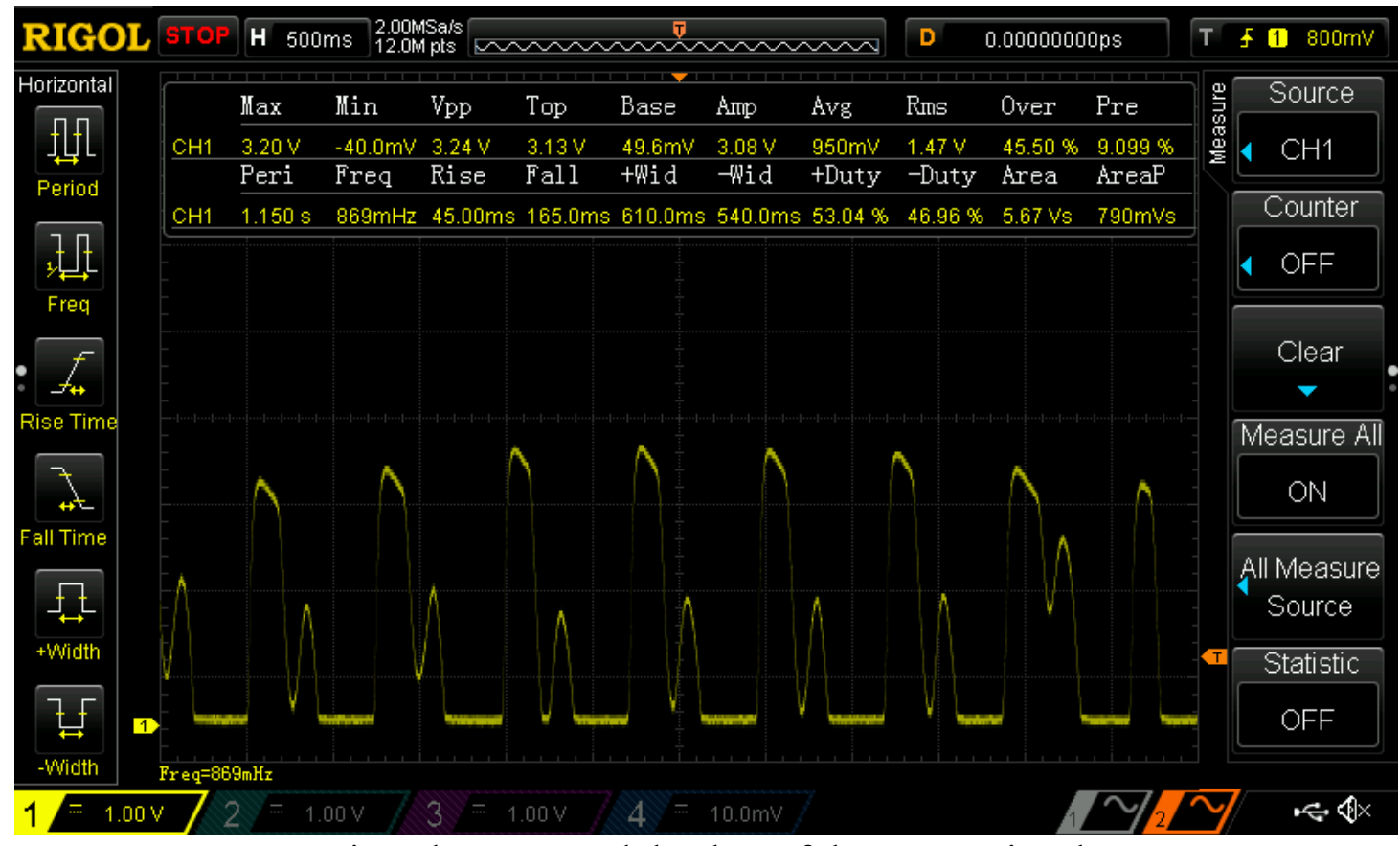

Fig.7 the wave and the data of the output signal

\section{Equations}

The equation of the project is:

错误!未找到引用源。

错误!未找到引用源。

And the gain of amplifier of the circuit:

错误!未找到引用源。

\section{Software}

Why use MAX7219?

Max7219 has these feature:

1.150uA Low-Power Shutdown:

Beneficial to the circuit.

2. Individual LED Segment Control:

It can control each dot of $8 * 8 \mathrm{LED}$, which is able to display LED dot accurately.

3. Display Blanked on Power-Up:

Protect circuit. 
Software design

Pulse measuring algorithm

1. The process of displaying the pulse waveform on LED:

(1)First, define data[8], turn all the dots on and then begin to read the information from circuit to change its elements.

(2)In the while(1) loop, define a float number voltage_info, which equates to the information.read(): information.read ()$=($ the real magnitude of voltage $) / 3.3$. Every loops we will read the analog signal delivered by the circuit.

(3)Using the if statement to decide the value of data[0] according to the value of voltage_info .it will turn on the corresponding dot on the first row of LED

(4) Perform the data presents on LED screen. Use this function:

void pattern_to_displaytwo

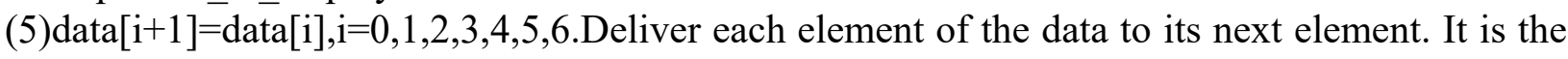
step that make the waveform dynamic, after $80 \mathrm{~ms}$, the pulse waveform presents on LED will change.

(6) wait_ms $(80)$, the frequency of collect the data.

(7)Go back and do next loop.

2. The process of calculating and displaying the pulse rate:

(1)We defined \#include $<$ time.h $>$, it is essential for us to use timer to count the period of the pulse. Timer is essential to count time. Then we defined interruptIn to trigger an event.

(2)When the voltage begin to increase, which means that, analogin_in.rise, this event happens and call the start function. Then, judge is more than one, which means that next if statement is satisfied. t.start(), it is able to count the period. And break this while loop.

(3)Then, when the next analogin_in.rise, the pulse have finish a period. We call the period_number to read the time of a period of the pulse. Then use an "if statement" to jump this while loop.

(4)Because the t.read() return a float number and the units are second(s). So frequency $=60 /$ period. Tenth, once are decade and unit, they are calculated by the frequency

(5)num is an array whose every four data presents a number, they presents number $0,1,2 \ldots 9$. According to the value of tenth and once ,A,B will equate to the corresponding elements in num.

(6)The next while loop can let the number rolling on the LED. pattern_to_display is the function that display the number on the LED.

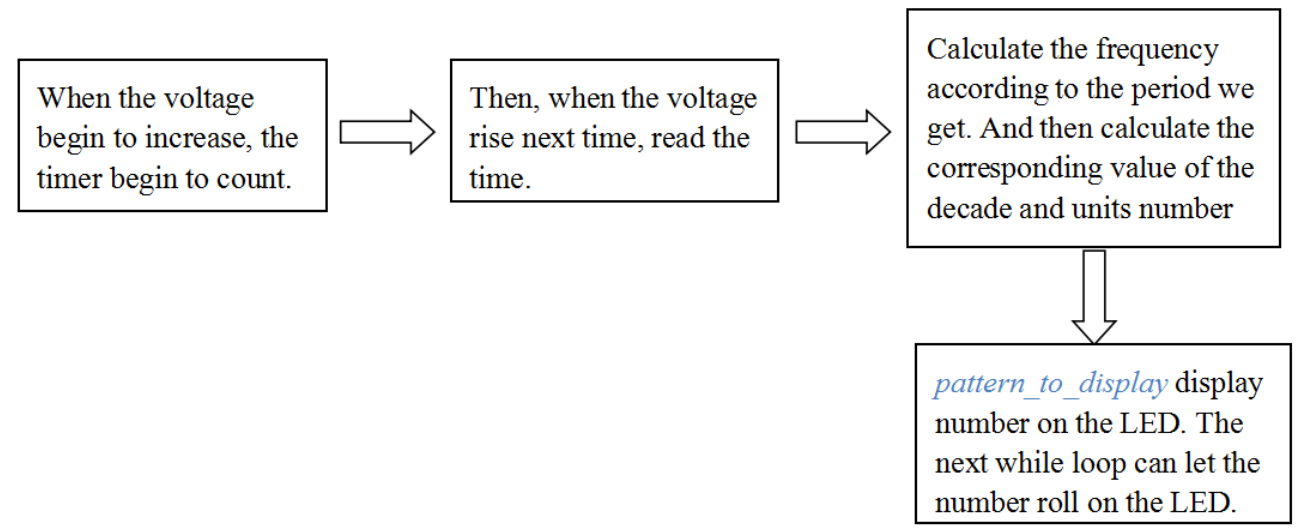

Fig. 8 flowchart of calculation 


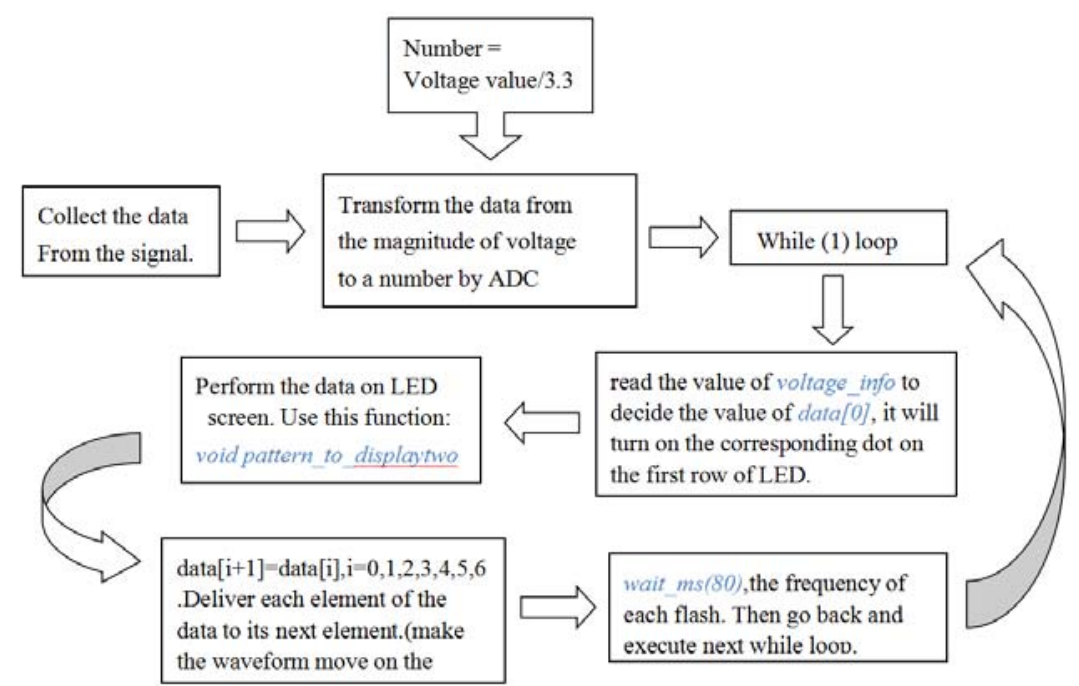

Fig.9 flowchart of displaying the pulse waveform

\section{Result}

At last. The circuit on the breadboard can be used to measure our pulse and amplify and filter the signal, so that the output analog signal can be transfer to the mbed which can use the MAX7219 to display the signal on the Nixie.

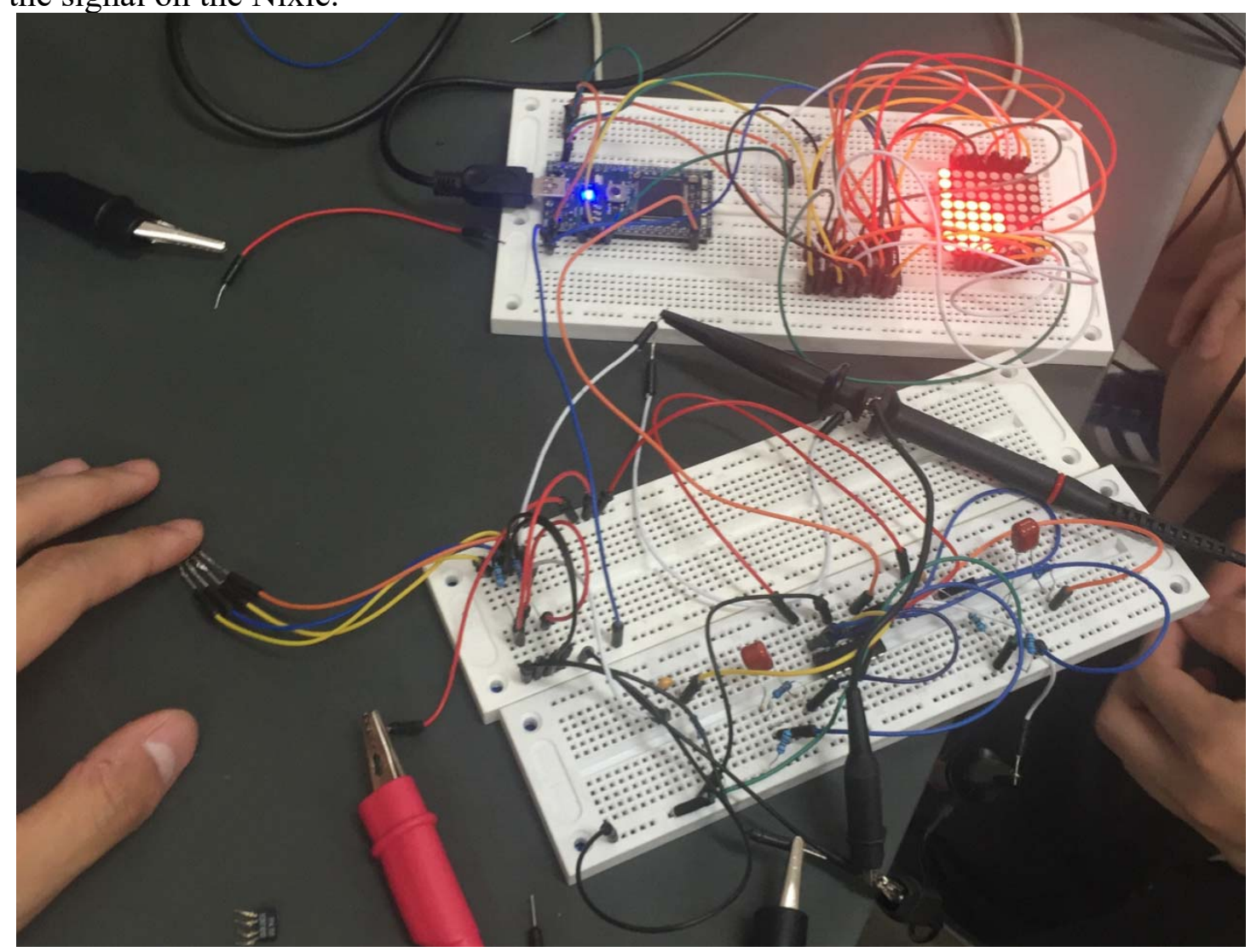

Fig.10 the shape of pulse shown on the screen

The screen is shown the shape of pulse, it' s stable and clear with the circuit. 


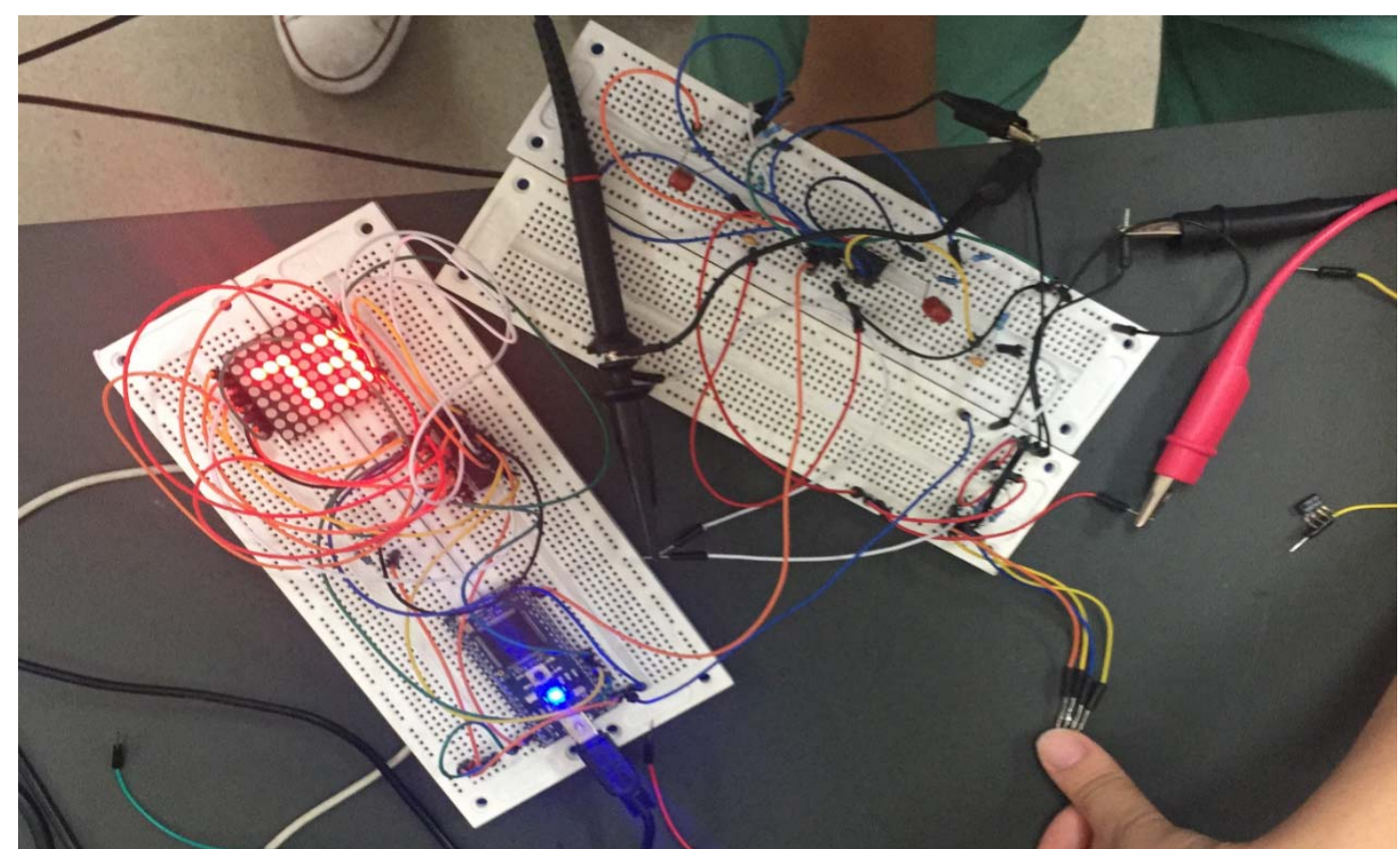

Fig. 11 the pulse rate is shown on the screen

As the graph shown, the Nixie screen can show the shape of the pulse and the rate of the pulse. The whole circuit and mbed worked as planned. However, the pulse is not stable because the output signal of the sensor is influenced by the press and light.

Mbed layout:

The Mbed is drawn with the software Altium Designer. And the model of the mbed and MAX7219 can be download from the internet. The finally result of PCB is shown below:

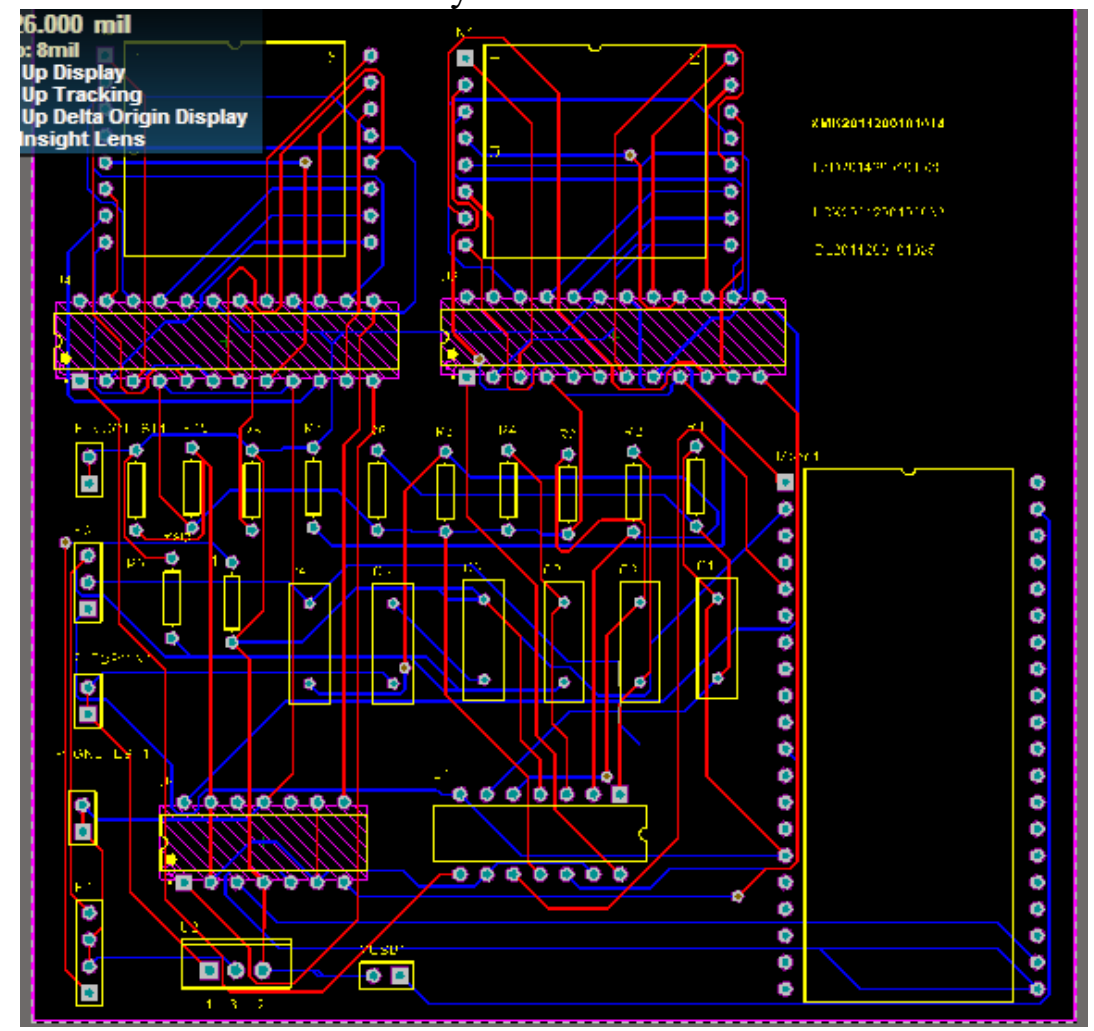

Fig.12 the PCB layout

\section{Analysis and Discussion}

In this project, there are a shortcoming that the bottom of the wave is cut off. About this situation, it need a reference voltage about $2 \mathrm{~V}$ that pull up the voltage that can display the whole shape of pulse. 
Another problem is about the code. The code which can display the ratio of pulse that cannot detect the ratio accurately, because of the influence from the noise, the mbed cannot detect the rising edge of the signal is not accurate. So the number is not stable sometimes.

\section{Conclusions}

After several test and the simulation on the breadboard, the 8x8 Nixie tube and its driver MAX7219 can be used to show the shape of the pulse and the ratio of the pulse, which means the project has been finished completely.

In this experiment, it is successful in getting relatively proper signal from the analogue circuits, which was used to filter and amplify the signals from the optical sensors. In order to show the data and waves shapes on the LED screen, an mbed (LPC 1768) was used to receive the analogue signals and transfer them to digital signals. Digital signals were used to be showed on screen by processing algorithm, which was also used to perform the shapes of waves. However, the precise shapes were hard to get given that the detecting speeds were not fast enough. The shapes got from the analogue circuits were hard to match the real shapes of heart rates because the filtering circuits' datasheet could not be matched properly. From the whole experiment, the essential part is how to get analogue signals from the optical sensors and how to transfer the analogue information into digital information. Hardware was used for collecting the real information and software was used to display the information on the screen.

Outcomes of our projects were relatively correctly matched with the original circuit. Errors could be easily seen. First of all, the shapes on the oscillator are not perfectly matched with the real heat beat, which is a result of improper amplifying gains and filtering parameters. Secondly, the shapes displayed on the LED screen are not matched with the original analogue signals. Detecting speeds and the algorithms should take the responsibility for that, which means that what we detected from the analogue signals lags off the real signals. Faster detecting speed and better LED screen are needed for better performance. In this experiment, only part of fingers can be used to test the heart beats, which means that analogue circuits need improvements. The display of heart beat speed was unexpectedly difficult because how to detect the raising edges and how to calculate the speed were difficult.

\section{Suggestions for further work}

Further work about the project is about the PCB layout, we have already draw the PCB with the software Altium Designer, after the PCB is printed, the component should be welded on the PCB and also connect to the battery. Check whether it works or not on the PCB.

The experiment can be extended in many ways. Optimized ways of amplifying and filtering were still under research. Furthermore, programming files and algorithm can be obviously developed, which are essential to the display sections. In the experiment, a situation was found that only some figures of the members can be tested, which means that there are problems of the analogue, which could not meet higher requirements and more general situations. So amplifying gains and optical sensors can be further developed.

\section{Acknowledgements}

I am very grateful to my team for their support during the stressful time while I was writing this report.

Thanks for the whole team, and we did pretty well together and finish the project smoothly, everyone in the team has try their best.

\section{References}

[1] Iii F J D, Gay D H. Biological inspiration for technological innovation[J]. IFAC Proceedings Volumes, 2013, 46(7):1-4.

[2] Xu S, Sun H, Li B, et al. Wide-Area Robust Decentralized Coordinated Control of HVDC Power System Based on Polytopic System Theory[J]. Mathematical Problems in Engineering, 2015, 2015(4):1-9.

[3] Urriza J M, Paez F E, Orozco J D, et al. Computational Cost Reduction for Real-Time Schedulability Tests Algorithms[J]. IEEE Latin America Transactions, 2015, 13(12):3714-3723.

[4] Nogueira B, Maciel P, Tavares E, et al. A Formal Model for Performance and Energy Evaluation of Embedded Systems[J]. EURASIP Journal on Embedded Systems, 2011, 2011(1):1-12.

[5] Average O. Optimization of an Accelerometer and Gyroscope-Based Fall Detection Algorithm[J]. Journal of Sensors, 2015, 2015:1-8. 